\title{
Zum Einfluss von Nachfrageverteilungen auf das Verhalten von Lagerhaltungsstrategien
}

\author{
SONJA KUHNT \& WIEBKE SIEBEN \\ FAKULTÄT STATISTIK \\ TECHNISCHE UNIVERSITÄT DORTMUND \\ 44221 DORTMUND
}

Lagerhaltungsstrategien beinhalten Anweisungen zur Berechnung von Lagerbeständen und Bestellmengen. In diesem Artikel wird der Effekt von Nachfrageverteilungen auf das Ergebnis von verschiedenen bekannten Strategien untersucht und ein Vergleich bezüglich erreichter Servicegrade durchgeführt. Als eine Folgerung aus diesem Vergleich wird ein nicht parametrisches Nachfragemodell als Basis für Lagerhaltungsstrategien vorgeschlagen.

Keywords: Lagerhaltungsstrategien, nicht parametrisches Nachfragemodell, Anwendungen in der Logistik

\section{Einleitung}

Lagerhaltungsstrategien dienen der Festsetzung der benötigten Höhe des Lagerbestandes, um zukünftige Nachfrage bestmöglich befriedigen zu können. Finanzielle Aspekte sind dabei genauso relevant wie die Notwendigkeit der Bereitstellung eines guten Service. Für die grundlegenden Konzepte in diesem Bereich siehe [Silv. 1998] oder [Temp. 2006] und die Literaturverweise darin.

Wir beschränken unsere Untersuchungen auf die Nachfrage einzelner Artikel in einem ,single-item singlelevel“-Rahmen mit periodischer Überprüfung des Lagerbestands und sogenannten „order-up-to“Bestellstrategien. D.h., der Lagerbestand wird am Ende jeder Periode aktualisiert und mit Hilfe einer Lagerhaltungsstrategie wird die Entscheidung getroffen, ob das Lager bis zu einer Bestellgrenze $S$ aufgefüllt werden soll (vgl. Abb. 1).

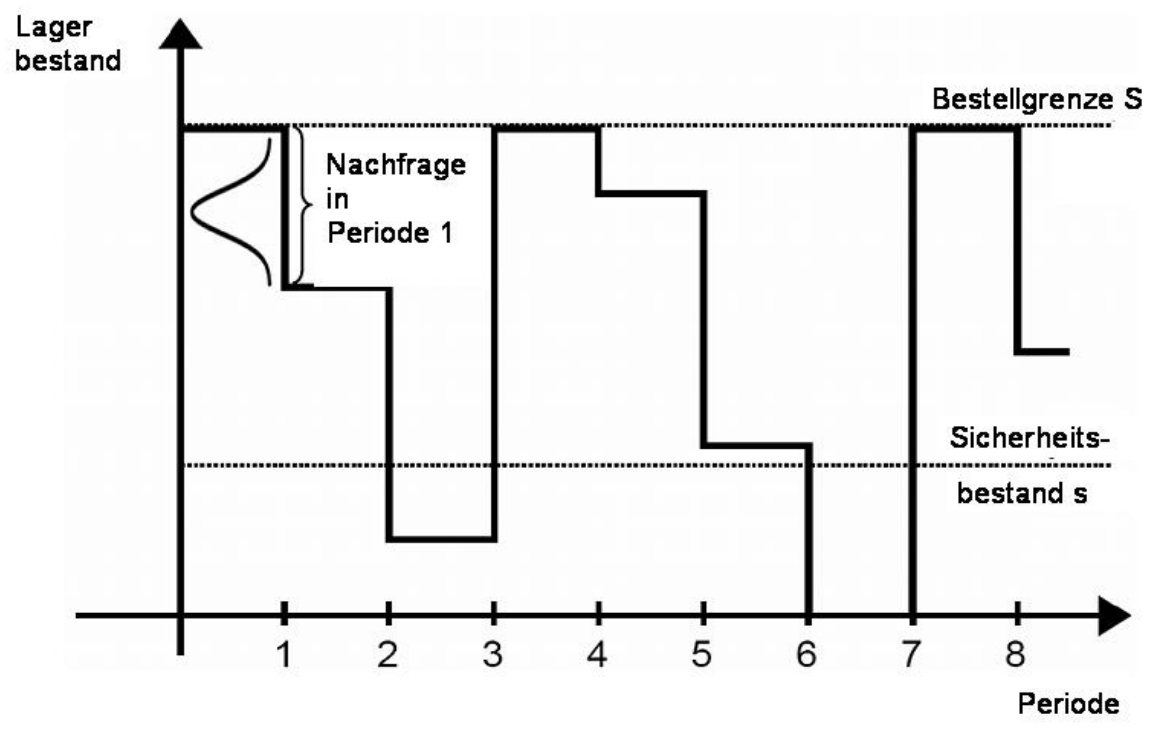

Abbildung 1: Setup

Einhergehend mit weitreichenden Veröffentlichungen existieren viele verschiedene Lagerhaltungsstrategien, welche oft sehr spezifisch und aufwendig sind, unter Berücksichtigung zahlreicher Variablen und Bedingungen. 
Die meisten Strategien basieren auf Annahmen, die die Nachfrage betreffen, zum Beispiel wird üblicherweise vorausgesetzt, dass die Nachfrage unabhängig und identisch normalverteilt ist - für gewöhnlich, ohne dass diese Annahme in der Praxis überprüft wird.

Das Ziel dieser Studie ist es, den Effekt von gültigen und verletzten Modellannahmen bezüglich der Nachfrage auf das Verhalten von Lagerhaltungsstrategien aufzuzeigen. Um rein diesen Effekt zu beobachten, wird eine Wiederbeschaffungszeit von null verwendet, d.h. das Lager kann sofort wieder aufgefüllt werden. Dies ist natürlich kein realistisches Szenario, da die Wiederbeschaffung in der Regel eine gewisse (zufällige) Zeit in Anspruch nimmt, genügt aber für den Zweck der Untersuchung.

Erste stochastische Ansätze bezüglich des optimalen Lagerbestands und der Modellierung von Lagerhaltungssituationen wurden in den Fünfzigerjahren des letzten Jahrhunderts vorgeschlagen. Arrow et al. [Arro. 1951] entwickeln eine optimale $(S, S)$-Strategie für ein statisches und ein dynamisches Lagerhaltungsmodell. Beide Modelle basieren auf Annahmen bezüglich der Nachfrageverteilung und berücksichtigen Kosten für Fehlmengen. Im dynamischen Modell wird der Lagerbestand als Markovkette betrachtet. Kurz darauf, im Jahr 1953 modellierten Dvoretzky et al. [Dvor. 1953] die Lagerhaltungssituation als Zwei-Personen Nullsummenspiel mit Verlustfunktionen und Entscheidungsregeln. Bezüglich der Optimalität von Lagerhaltungsstrategien zeigt Scarf [Scar. 1960] auf, dass bei linearen Lager- und Fehlmengenkosten eine optimale Strategie immer vom ( $s, S)$-Typ ist. Basierend auf diesen Erkenntnissen entwickelt Iglehart [Igle. 1963] eine approximativ optimale Strategie für einen unendlichen Planungshorizont.

Heutzutage steht die Optimalität von Bestellstrategien für Lagerhaltungsmodelle im Fokus vieler Arbeiten. Die betrachteten Modelle sind dabei zumeist auf spezielle Situationen und Annahmen zugeschnitten. Cetinkaya und Parlar [Ceti. 2004] schlagen eine optimale stationäre Politik für ein Lagerhaltungsmodell mit periodischer Überwachung, unabhängig und identisch verteilter Nachfrage, nichtlinearen Fehlmengenkosten und endlichem Planungshorizont vor. Gallego und $\mathrm{Hu}$ [Gall. 2004] stellen eine optimale Politik für den endlichen sowie den unendlichen Planungshorizont für ein Lagerhaltungsmodell mit periodischer Überwachung und begrenzter Lager- bzw. Produktionskapazität vor, in dem Nachfrage und Wiederbeschaffung jeweils einer Markovkette entspringen. Hollier, Mak und Lai [Holl. 2002] entwickeln eine optimale ( $s, S)$-Politik für ein Modell mit kontinuierlicher Überwachung und gemischt-poissonverteilter Nachfrage, in dem die Nachfrage ab einer gewissen Größe, und falls der Lagerbestand unterhalb eines kritischen Wertes liegt, direkt in eine Nachschubbestellung umgesetzt wird, die den Lagerbestand auf die Bestellgrenze $\mathrm{S}$ auffüllt, während die übrige Nachfrage aus dem vorhandenen Lagerbestand bedient wird.

Einige Ansätze basieren auf dem deterministischen „Economic Order Quantity“ (EOQ) Modell [Harr. 1915]. Yu [Yu 1995] entwickelt ein robustes EOQ-Modell, in dem Nachfrage, Bestell- und Lagerhaltungskosten als Zufallsvariablen betrachtet werden. Presman und Sethi [Pres. 2004] stellen eine Politik für ein Modell mit kontinuierlicher Überwachung und einer Nachfrageverteilung vor, die sich aus einer deterministischen und einer stochastischen Komponente zusammensetzt. Diese Politik entspricht der EOQ-Formel, wenn die stochastische Komponente der Nachfrage fehlt.

Bei anderen Ansätzen werden die Abweichungen der Nachfrageprognosen von den tatsächlich eingetretenen Nachfragen in die Lagerhaltungsstrategie mit einbezogen. Heath und Jackson [Heat. 1994] stellen ein Modell für die Veränderung der Nachfragevorhersage vor, das sie „Martingale Model of Forecast Evolution“ nennen. Toktay und Wein [Tokt. 2001] setzen diese Idee für ein Lagerhaltungsmodell mit Kapazitätsbeschränkung um. Eine nichtparametrische Umsetzung liefert Abels [Abel. 1999], der eine Lagerhaltungsstrategie vorschlägt, bei der die Nachfrageprognose um die obere Konfidenzgrenze für den Median der Prognosefehler ergänzt wird.

Offenbar gibt es eine Vielfalt an Lagerhaltungsmodellen und Strategien. In der Praxis und speziell in großen Lagern mit vielen Artikeln könne Modellannahmen und Nachfrageverteilungen für jeden einzelnen Artikel aufgrund des dafür nötigen Aufwands und der entstehenden Kosten nicht überprüft werden. Daher ist es von besonderer Bedeutung, praxisnahe Lagerhaltungspolitiken mit wenigen Modellannahmen hinsichtlich des Servicegrades zu untersuchen und auch in Situationen zu vergleichen, in denen diese wenigen Annahmen verletzt sind. Generell wird eine Lagerhaltungspolitik benötigt, die vielen potentiell unterliegenden Nachfrageverteilungen gerecht wird. In diesem Artikel werden Lagerhaltungsmodelle und Bestellpolitiken hinsichtlich ihrer Ergebnisse in simulierten und realen Lagerhaltungssituationen verglichen. Zudem wird der Einfluss der Häufigkeit von Perioden ohne Nachfrage, die ein bekanntes Problem in der Lagerhaltungspraxis darstellen, auf die erreichten Servicegrade untersucht.

Der Artikel beginnt mit einer Diskussion des Servicegrades als Maß für guten Service (Abschnitt 2). In Abschnitt 3 schließt sich eine Beschreibung von sechs Lagerhaltungsmodellen mit deren Bestellstrategien an. In Abschnitt 4 werden simulierte Lagerhaltungssituationen und reale Daten mit einem beachtlichen Anteil an Nullnachfrage-Perioden beschrieben. In Abschnitt 5 werden die Lagerhaltungspolitiken angewendet und Unterschiede zwischen Ziel-Servicegrad und erreichtem Servicegrad verglichen. Abschnitt 6 gibt eine Zusammenfassung und Diskussion der Ergebnisse. 


\section{Servicegrade}

Für den Vergleich verschiedener Lagerhaltungsstrategien anhand ihrer Lieferbereitschaft in verschiedenen Bestandssituationen wird ein den Service messendes Kriterium benötigt. Drei gängige Maßzahlen bezüglich des Servicegrades sind der $\alpha$-, $\beta$ - und der $\gamma$-Servicegrad.

Allgemein messen Servicegrade die Lieferbereitschaft nachgefragter Güter. Sie spiegeln die Relationen von Nachfrage, befriedigter Nachfrage und nicht befriedigter Nachfrage wider. Somit handelt es sich um bedeutende Hilfsmittel zum Vergleich verschiedener Lagerhaltungsstrategien. Die Größen werden für unbekannte theoretische Wahrscheinlichkeiten von Fehlbeständen in Abhängigkeit des unterstellten zugrunde liegenden Lagerhaltungsmodells geschätzt.

Als erreichter $\alpha$-Servicegrad wird der empirische Anteil der Perioden mit vollständig befriedigter Nachfrage an allen Perioden bezeichnet. Der erreichte $\beta$-Servicegrad ist der empirische Anteil der befriedigten Nachfrage in einer Periode an der gesamten Nachfrage der Periode. Der $\beta$-Servicegrad liefert also Informationen über die Höhe der aufgetretenen Fehlmengen, während der $\alpha$-Servicegrad nur berücksichtigt, ob Fehlbestände aufgetreten sind oder nicht.

Für den $\gamma$-Servicegrad, eine dritte Maßzahl für den Service, existieren verschiedene Definitionen. Hierfür wird nicht nur die Höhe der fehlenden Mengen gemessen, sondern auch die Zeit, in der keine Belieferung möglich ist. Im Falle entgangener Verkäufe, d.h. ohne Lieferrückstände, und einer Wiederbeschaffungszeit der Länge Null, stimmen der $\gamma$-Servicegrad und der $\beta$-Servicegrad überein. Diese Annahmen werden im Folgenden als erfüllt angesehen. Für weitere Details zu diesen Servicemaßen siehe [Schn. 1981].

Da jede Periode ohne Nachfrage eine Periode mit vollständig befriedigter Nachfrage ist, erreicht ein Artikel mit 80 Prozent Nullnachfrage-Perioden einen $\alpha$-Servicegrad von 80 Prozent, wenn er nicht auf Lager gehalten wird, d.h. wenn der Lagerbestand für diesen Artikel immer gleich Null ist. Wenn der Artikel auf Lager ist, d.h. wenn der Lagerbestand größer als Null ist, erreicht der $\alpha$-Servicegrad daher mindestens 80 Prozent. Bei dem Ziel, einen $\alpha$-Servicegrad von 90 Prozent zu erreichen, ist die mögliche Differenz zwischen dem erreichten $\alpha$ Servicegrad und dem vorgegebenen $\alpha$-Servicegrad für einen Artikel mit vielen Perioden mit Nullnachfrage kleiner als für einen Artikel mit wenigen Nullnachfrage-Perioden. Trotzdem ist der $\alpha$-Servicegrad ein hilfreiches Werkzeug um die Lieferfähigkeit nachgefragter Güter zu beurteilen. Ein Artikel der nur einmal jährlich nachgefragt wird, muss nicht auf Lager sein, sondern kann mit einer gewissen Wiederbeschaffungszeit bestellt werden, wenn er benötigt wird. Die Konsequenzen von Fehlbeständen müssen gegen die Kosten und Bemühungen abgewogen werden, die die Lagerhaltung eines bestimmten Artikels mit sich bringt. Wenn die sofortige Befriedigung der Nachfrage als zwingend erforderlich angesehen wird, kann der geforderte $\alpha$ Servicegrad so hoch gesetzt werden, dass er durch die Perioden ohne Nachfrage allein nicht erreicht werden kann.

Im Gegensatz zum $\alpha$-Servicegrad berücksichtigt der $\beta$-Servicegrad Perioden ohne Nachfrage nicht. Trotzdem ist die Erfassung der Fähigkeit zur Befriedigung der Nachfrage nicht besser geeignet als der $\alpha$-Servicegrad. Wenn ein Artikel nur einmal jährlich bestellt wird und daher nicht auf Lager ist, kann keine Nachfrage, gleich welcher Höhe, in derselben Periode gedeckt werden, in der die Nachfrage auftritt. In diesem Beispiel ist der erreichte $\beta$ Servicegrad für ein ganzes Jahr gleich Null. Dies entspricht nicht dem Umstand, dass die Bestandshöhe von Null die meiste Zeit über angemessen ist. Aus diesem Grunde wird das $\alpha$-Servicegrad als Maß für die Fähigkeit, die Nachfrage zu decken, verwendet.

\section{Lagerhaltungsstrategien}

Lagerhaltungsmodelle basieren auf Annahmen bzgl. der vorliegenden Lagerhaltungssituation. Unter Verwendung dieser Modelle sind Lagerhaltungsstrategien als Vorschriften aufzufassen, wie optimale Lagerbestände oder Bestellmengen zu berechnen sind. Eine optimale Strategie kann anhand von Kosten- oder Servicegradüberlegungen gefunden werden. Diese zwei Ansätze sind häufig äquivalent und beide lassen Raum für die Subjektivität des Anwenders, da üblicherweise nicht alle Kosten bekannt sind und geschätzt werden müssen. Somit gibt es keinen objektiven Weg einen angemessenen Soll-Servicegrad zu bestimmen.

Nachfolgend werden sechs verschiedene $(1, S)$-Lagerhaltungsstrategien als Spezialfälle der (s,S)-Strategien diskutiert. Zwei dieser sechs Strategien beinhalten feste Sicherheits- und Grundbestände, auf die das Lager zu Beginn jeder Periode aufgefüllt wird. Die erste Lagerhaltungsstrategie „P1“ basiert auf der Annahme einer u.i.v. normalverteilten Nachfrage. Für die zweite Strategie „P2“ wird eine u.i.v. Gamma-Verteilung unterstellt. Die Lagerhaltungsstrategien „P3“ und „P4“ haben feste Sicherheitsbestände, aber die Grundbestände werden in jeder Periode aktualisiert. Dazu wird basierend auf vergangenen Beobachtungen und der aktuellen Nachfrage eine Ein-Schritt-Prognose eines autoregressiven integrierten Moving-Average-Modells (ARIMA) verwendet. Die festen Höhen der Sicherheitsbestände werden mittels der Varianz des Weißen Rauschens (P3) bzw. der $\alpha$ - 
Quantile der Fehlmengen bestimmt, die auftreten, wenn kein Sicherheitsbestand vorgehalten wird (P4). Die verbleibenden Lagerhaltungsstrategien „P5“ und „P6“ haben Sicherheits- und Grundbestände, die in jeder Periode aus der letzten Nachfrage berechnet werden. Für die Strategie P5 wird erneut eine u.i.v. normalverteilte Nachfrage angenommen, aber im Gegensatz zu P1 werden der Sicherheitsbestand und die Bestellgrenze in jeder Periode neu berechnet. Bei der Strategie P6 wird die empirische Verteilungsfunktion als Schätzung für die unbekannte Verteilungsfunktion verwendet, die in jeder Periode aktualisiert wird. In allen diesen Strategien wird die Wiederbeschaffungszeit auf Null gesetzt, und statt Kosten- nur Servicegradbedingungen berücksichtigt. Ob Lieferrückstände zugelassen werden, ist daher nicht relevant.

\subsection{Strategien mit festen Grund- und Sicherheitsbeständen}

Bei den Lagerhaltungsstrategien P1 und P2 handelt es sich um parametrische Strategien, die zur Berechnung der optimalen Bestellgrenze $S^{*}$ recht einfache Formeln bieten. Wie im statischen AHM-Modell [Arro. 1951] wird angenommen, dass die Nachfrage $x_{t}$ zu jedem Zeitpunkt $t$ unabhängig identisch verteilt ist mit Verteilungsfunktion $F$. Hier wird $S^{*}$ nicht durch Kostenminimierung bestimmt, sondern durch die Vorgabe eines zu erreichenden Servicegrads $\alpha$. Damit ist $S^{*}$ die optimale Bestellgrenze, die folgende Gleichung erfüllt:

$$
P\left(x_{t} \leq S^{*}\right)=F\left(S^{*}\right)=\alpha
$$

Im Fall von P1 wird eine normalverteilte Nachfrage mit Erwartungswert $\mu$ und Varianz $\sigma^{2}$ angenommen, so dass sich $S^{*}$ schreiben lässt als

$$
S^{*}=\Phi^{-1}(\alpha) \cdot \sigma+\mu
$$

wobei $\Phi$ die Verteilungsfunktion der Standardnormalverteilung bezeichnet. Diese Bestellgrenze kann aufgeteilt werden in den Grundbestand $\mu$ und den Sicherheitsbestand $\Phi^{-1}(\alpha) \cdot \sigma$. Dies ist die in der Literatur übliche Darstellung.

Im Fall von P2 ist $F$ die Verteilungsfunktion einer Gammaverteilung und $S^{*}$ ist gleich dem $\alpha$-Quantil von $F$. Die Bestellgrenze $S^{*}$ kann aufgeteilt werden in den Grundbestand, der durch den Median $\widetilde{x}_{0.5}$ gegeben ist, und in den Sicherheitsbestand $S^{*}-\widetilde{x}_{0.5}$.

\subsection{Strategien mit festen Sicherheitsbeständen und angepassten Grundbeständen}

Die Annahme der unabhängigen Nachfrage stellt sich oft als eher unrealistisch heraus. In einem solchen Fall kann z.B. eine korrelierte Nachfrage mithilfe eines ARIMA-Modells (P3 und P4) modelliert werden. Auch diese Strategien sind parametrisch.

Wenn der zugrunde liegende Prozess der korrelierten Nachfrage ein ARIMA-Prozess ist, kann die Nachfrage $x_{t}$ in der Periode $t$ beschrieben werden durch

$$
\left(1-\sum_{j=1}^{p} \varphi_{j} B^{j}\right)(1-B)^{d}\left(x_{t}-\mu\right)=\left(1-\sum_{j=1}^{p} \theta_{j} B^{j}\right) \cdot \varepsilon_{t}
$$

wobei $\theta_{1}, \ldots, \theta_{q}, \varphi_{1}, \ldots, \varphi_{p}$ Konstanten, $B$ der Backshift-Operator, $\mu$ der Erwartungswert und $\varepsilon_{t}$ Weißes Rauschen mit Varianz $\sigma_{\varepsilon}^{2}$ ist. Unter gewissen Voraussetzungen ist die Bestellgrenze

$$
S_{t}^{*}=\hat{x}_{t}+\Phi^{-1}(\alpha) \cdot \sigma_{\varepsilon}
$$


optimal [John. 1975], wobei $\hat{x}_{t}=E\left(x_{t} \mid x_{t-1}, \ldots, x_{1}, \hat{\varepsilon}_{t-1}, \ldots, \hat{\varepsilon}_{1}\right)$. Folglich ist die optimale Bestellgrenze $S_{t}^{*}$ in Periode $t$ bei der Lagerhaltungsstrategie P3 gegeben durch die Summe aus der Ein-Schritt-Prognose $\hat{x}_{t}$ und dem $\alpha$-Quantil der Verteilung von $\varepsilon_{t}$. Hier kann $S_{t}^{*}$ aufgeteilt werden in den Grundbestand $\hat{x}_{t}$ und den Sicherheitsbestand $\Phi^{-1}(\alpha) \cdot \sigma_{\varepsilon}$.

Der Grundbestand der Lagerhaltungsstrategie P4 wird berechnet wie bei Strategie P3, wohingegen der Sicherheitsbestand gegeben ist durch das empirische $\alpha$-Quantil der Verteilung der Fehlmengen [Abel. 1999], die auftreten, wenn nur die Ein-Schritt-Prognose vorgehalten wird.

\subsection{Strategien mit angepassten Grund- und Sicherheitsbeständen}

Strategien mit angepassten Grund- und Sicherheitsbeständen nutzen alle zur Verfügung stehenden Informationen um über die Bestellmenge zu entscheiden: In jeder Periode werden die Schätzer unter Verwendung der letzten beobachteten Nachfrage neu berechnet.

Für die folgenden Strategien wird angenommen, dass die Nachfrage unabhängig und identisch verteilt ist mit Verteilungsfunktion $F$, wie zuvor auch bei P1 und P2. Die optimale Bestellgrenze $S^{*}$ für einen gegebenen Zielservicegrad $\alpha$ ist dann erneut gegeben durch

$$
P\left(x_{t} \leq S^{*}\right)=F\left(S^{*}\right)=\alpha \Leftrightarrow S^{*}=F^{-1}(\alpha) .
$$

Strategie P5 ist eine Update-Version von Strategie P1, basierend auf der Annahme der Normalverteilung. Der Basisbestand $\mu_{t}$ in Periode $t$ wird durch $\bar{x}_{t}$, der mittleren Nachfrage während der Perioden 1 bis $t$-1, geschätzt. Die Schätzung des Sicherheitsbestands erfolgt durch $\Phi^{-1}(\alpha) \cdot \hat{\sigma}_{t}$, d.h. durch das Produkt des $\alpha$-Quantils einer Standardnormalverteilung und der aktualisierten geschätzten Standardabweichung, die gemeinsam die optimale Bestellgrenze $S_{t}^{*}$ in Periode $t$ darstellen.

Als Alternative zu den im Vorherigen beschriebenen parametrischen Lagerhaltungsstrategien führen wir nun eine nichtparametrische Strategie (P6) ein, die auf der Annahme der unabhängig identisch verteilten Nachfrage beruht. Die unbekannte Verteilung der Nachfrage wird durch die empirische Verteilungsfunktion geschätzt. Die Bestellgrenze $S_{t}^{*}$ in Periode $t$ kann in den Grundbestand $\widetilde{x}_{0.5, t}$, also den aktualisierten Median, und den Sicherheitsbestand $\widetilde{x}_{\alpha, t}-\widetilde{x}_{0.5, t}$, der Differenz zwischen $S_{t}^{*}$ und dem Median, aufgeteilt werden.

Bei diesen beiden Strategien wird das Lager zu Beginn einer jeden Periode auf die optimale Bestellgrenze $S_{t}^{*}$ aufgefüllt, es sei denn, die Bestandshöhe am Ende der vorigen Periode ist größer als $S_{t}^{*}$.

Es ist ebenso möglich, zur Schätzung von $F_{t}^{-1}$ nur eine feste Anzahl der jüngsten Beobachtungen der Nachfrage zu verwenden. Das $\alpha$-Quantil der empirischen Nachfrage wird dann in einem gleitenden Fenster bestimmt.

\section{Lagersituationen}

Die vorgestellten Lagerhaltungsstrategien basieren auf Annahmen bezüglich der Verteilung der Nachfrage. Der Effekt von verletzten Verteilungsannahmen kann in realen Lagersituationen nicht bestimmt werden, da die wahre Verteilung natürlich unbekannt ist. Wendet man die Lagerhaltungsstrategien auf reale und simulierte Lagersituationen an und vergleicht die erreichten Servicegrade, so lassen sich jedoch Aussagen darüber treffen, wie die Strategien auf Verletzungen der Modellannahmen reagieren. Unsere Analyse basiert auf folgenden simulierten Lagersituationen.

Zunächst werden die Daten der Nachfrage aus Normal- und Gammaverteilungen simuliert, da diese in der Praxis häufig angenommen werden. Die Parameter für die Simulation von 500 Datenreihen der Länge 52 aus einer Gammaverteilung wurden aus realen Lagersituationen geschätzt. Damit ist die Datenreihe der simulierten gammaverteilten Nachfrage einer wahren Nachfrage ähnlich, wenn die Annahme der Gammaverteilung zutrifft. Normalverteilungen sind symmetrisch um ihren Erwartungswert und negative Realisationen treten immer mit einer gewissen Wahrscheinlichkeit auf. Da die Nachfrage nicht negativ sein kann, werden die Parameter für die Simulation der normalverteilten Nachfrage so gewählt, dass eine negative Nachfrage nur mit einer kleinen 
Wahrscheinlichkeit generiert wird. Es werden 500 Datenreihen aus einer Normalverteilung mit Erwartungswert 1000 und zufälliger Varianz zwischen 1 und 250 simuliert.

Zusätzlich zu den simulierten Lagersituationen werden auch reale Lagersituationen betrachtet. Die Daten stammen aus einem Kohlebergbau-Unternehmen und enthalten Lagerinformationen über 11830 Posten. Sie enthalten unter anderem das Datum der Bedarfsäußerung, das gewünschte Lieferdatum und das Datum der tatsächlichen Auslieferung. Bei den Gütern handelt es sich vorwiegend um Ersatzteile, wie Schrauben und Schläuche, Farben und Lacke, Werkzeuge, Maschinen und Maschinenteile aber auch um Arbeitsbekleidung, Reinigungsmittel, Dieselöl, Sanitärobjekte, Feuerlöscher, Erste-Hilfe-Ausstattung, Körperpflegezubehör sowie Kaffee, Tee und Kekse. Die zur Verfügung stehenden Daten wurden über ein Jahr bezüglich des tatsächlichen Auslieferungsdatums aufgezeichnet, obwohl das Datum der gewünschten Auslieferung der vorrangig interessierenden Variable Nachfrage am nächsten kommt. Somit sind alle Lieferungen eines Jahres verfügbar, nicht aber alle gewünschten Lieferungen.

Aus den Daten ist nicht ersichtlich, ob die ausgelieferte Menge mit der angeforderten Menge übereinstimmt oder ob diese in Teillieferungen zugestellt wurde. Jedoch werden potentielle Teillieferungen vereinigt, indem alle ausgelieferten Mengen eines Artikels mit demselben gewünschten Lieferdatum zur Tagesnachfrage für diesen Artikel aufsummiert werden.

Es treten häufig Tage ohne Nachfrage auf. Dies betrifft vor allem die meisten Samstage und Sonntage. Wir setzen die Periodenlänge auf eine Woche fest, wodurch sich die Zahl von Perioden mit Nullnachfragen verglichen mit einer Periodenlänge von einem Tag reduziert. Diese Wahl erscheint vernünftig, da auftretende Nachfrage selten in der Woche ihres Auftretens befriedigt wird und Verzögerungen von bis zu zwei Jahren zu verzeichnen sind. Nichts desto trotz ist die Nachfrage ungeachtet der Periodenlänge unregelmäßig (vgl. Tabelle $1)$.

\begin{tabular}{ll}
\hline Häufigkeit Nullnachfrage & Anzahl Güter \\
\hline bis $10 \%$ & 616 \\
$10 \%-20 \%$ & 263 \\
$20 \%-30 \%$ & 298 \\
$30 \%-40 \%$ & 345 \\
$40 \%-50 \%$ & 376 \\
$50 \%-60 \%$ & 472 \\
$60 \%-70 \%$ & 723 \\
$70 \%-80 \%$ & 1056 \\
$80 \%-90 \%$ & 1701 \\
$90 \%-95 \%$ & 2060 \\
mind. $95 \%$ & 3920 \\
\hline
\end{tabular}

Tabelle 1: Häufigkeit von Nullnachfrage in Wochendaten der Nachfrage

Wir gruppieren die Artikel bezüglich des $\alpha$-Servicesgrades, der erreicht wird, wenn der Lagerbestand immer gleich Null gehalten wird, was identisch zur Häufigkeit der Nullnachfrage jedes Artikels ist. Die Anwendung von Bestellstrategien auf diese Gruppen könnte den Einfluss von Häufigkeiten von Nullnachfrage auf die erreichten Servicegrade aufdecken. Für Artikel, deren erstes Nachfragequartil größer als Null ist, ist in weniger als 25 Prozent der Perioden eine Nullnachfrage zu verzeichnen. In diese Kategorie fallen 1001 Güter, sie bilden die Gruppe 1. Die Gruppe 2 besteht aus allen Artikeln, deren erstes Quartil gleich Null, das zweite Quartil aber größer als Null ist. Diese Gruppe enthält 897 Artikel. Analog werden 1785 Artikel der Gruppe 3 zugeordnet. Ihre zweiten Quartile sind alle gleich Null, aber die dritten Quartile sind größer als Null. Die übrigen 8139 Artikel bilden die Gruppe 4. Für alle Artikel dieser Gruppe wurde in mindestens 75 Prozent der Perioden Nullnachfrage verzeichnet.

Den realen Nachfragedaten kann jedoch keins der in Kapitel 3 vorgestellten Lagermodelle allein zugrunde liegen. $\chi^{2}$-Anpassungstests führen zur Ablehnung der Hypothesen normal-, exponential-, poisson- und lognormalverteilter Nachfrage für alle oder zumindest fast alle Artikel. Nur für 588 Artikel aus Gruppe 1 konnte die Hypothese einer gammaverteilten Nachfrage nicht verworfen werden. Für die meisten dieser Artikel konnten jedoch auch ARIMA-Prozesse als zugrunde liegende Prozesse nicht ausgeschlossen werden. Die Plausibilität eines zugrunde liegenden ARIMA-Prozesses ist aufgrund der Datenmenge nur anhand von Tests auf korrelierte Residuen überprüft worden. Die meisten Testergebnisse weisen nicht darauf hin, dass die angepassten ARIMAModelle nicht geeignet wären.

\section{Leistungsfähigkeit der Lagerhaltungsstrategien}


Die meisten der beschriebenen Lagerhaltungsstrategien enthalten unbekannte Parameter, die vor der Anwendung der Strategien auf die Lagersituationen geschätzt werden müssen. Zur Schätzung der unbekannten Parameter in P1 und P2, wird die Nachfragereihe in zwei Hälften geteilt. Die notwendigen Parameter werden für jede Reihe aus der ersten Hälfte geschätzt. Danach werden die Strategien auf die zweite Hälfte der Nachfragereihe angewendet.

Für die Anwendung der Lagerhaltungsstrategien P3 und P4 auf die Daten müssen ARIMA-Modelle angepasst werden. Die Ordnung dieser Modelle, die Parameter $\theta_{1}, \ldots, \theta_{q}, \varphi_{1}, \ldots, \varphi_{p}$ und die Varianz $\sigma_{\varepsilon}$ sind unbekannt.

Für die Strategie P3 werden die Ordnung und Varianz aus der ersten Hälfte der Nachfragereihe geschätzt. Bei der Anwendung der Strategien werden die Parameter in jeder Periode der zweiten Hälfte aktualisiert, und es wird die Ein-Schritt-Prognose für die nächste Periode berechnet. Ist die resultierende Bestellgrenze kleiner als der Lagerbestand am Ende der vorigen Periode, wird nichts aufgefüllt. Für Politik P4 werden Modellordnung und Varianz aus dem ersten Viertel der Daten geschätzt. Dann werden die Parameterschätzungen jeder Periode im zweiten Viertel der Datenreihe aktualisiert und in der folgenden Periode wird die Ein-Schritt-Prognose verwendet. Die empirischen $\alpha$-Quantile der Fehlmengen im zweiten Viertel der Datenreihen bilden die Sicherheitsbestände. Strategie P4 wird dann auf die übrige zweite Hälfte der Nachfragereihen angewendet.

Für die Strategien P5 und P6 werden die Nachfragereihen halbiert, um Vergleichbarkeit sicherzustellen, obwohl keine Parameter aus den ersten Datenhälften geschätzt werden müssen. Wie die übrigen Strategien werden sie auf die zweiten Hälften der Reihen angewendet.

Die Lagerhaltungsstrategien werden auf die simulierten Nachfragereihen und die Nachfragereihen der Gruppen 1 bis 4 (jeweils der Größe 500) mit den Soll-Servicegraden 50, 70 und 90 Prozent angewendet. Die Strategien P3 und P4 unterscheiden sich in der Berechnung der Sicherheitsbestände, aber die Grundbestände werden auf die gleiche Art und Weise bestimmt. Ein Vergleich der Strategien P3 und P4 zeigt, dass mit P4 noch schlechtere Servicegrade erreicht werden als mit Strategie P3, weshalb Strategie P4 im Weiteren nicht weiter betrachtet werden soll. Die Unterschiede zwischen erreichten und Zielservicegraden werden mit Hilfe von Boxplots (vgl. Abb. 2, Abb. 3, Abb. 4) dargestellt. Im Idealfall ist der Median eines solchen Leistungs-Boxplots gleich Null und die Spannweite ist gering. Bei der Analyse der Abbildungen ist zu bedenken, dass die Strategien nur auf die Hälfte der Nachfragereihen angewendet werden und dass nicht jede zweite Hälfte der Nachfragereihen beispielsweise eines Gutes in Gruppe 4 einen Nullnachfrageanteil von mindestens 75 Prozent hat, wie es für die komplette Reihe der Fall ist. 
Ziel: $50 \%$

Nachfrage: normalverteilt

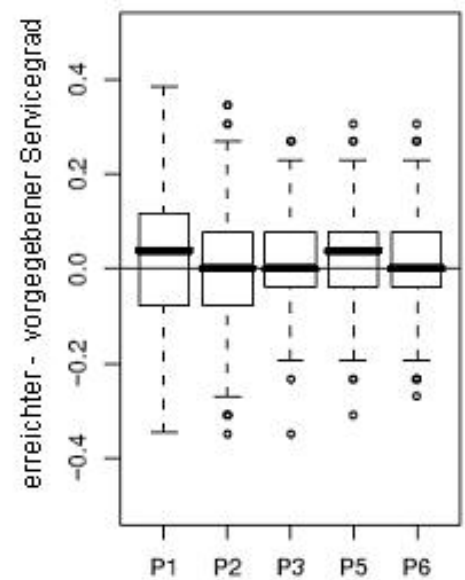

Ziel: $50 \%$

Nachfrage: gammaverteilt

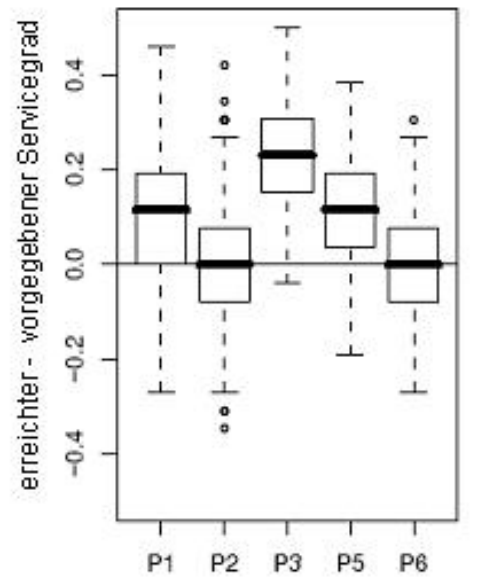

Ziel: $70 \%$

Nachfrage: normalverteilt

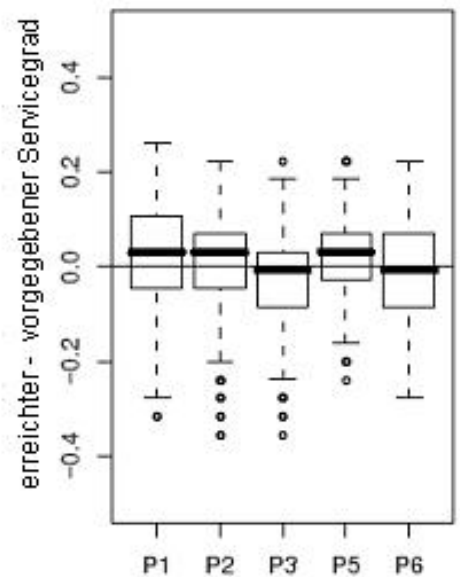

Ziel: $70 \%$

Nachfrage: gammaverteilt

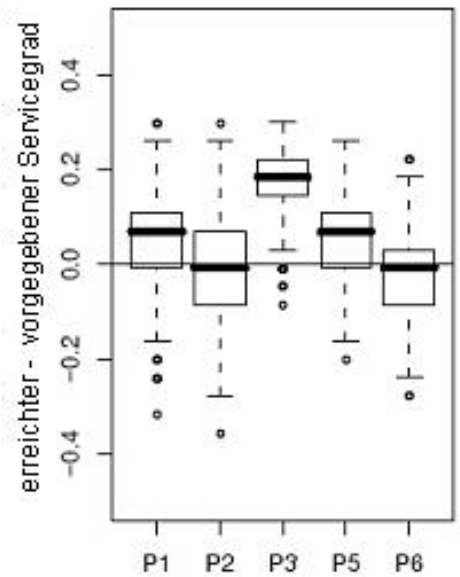

Ziel: $90 \%$

Nachfrage: normalverteilt

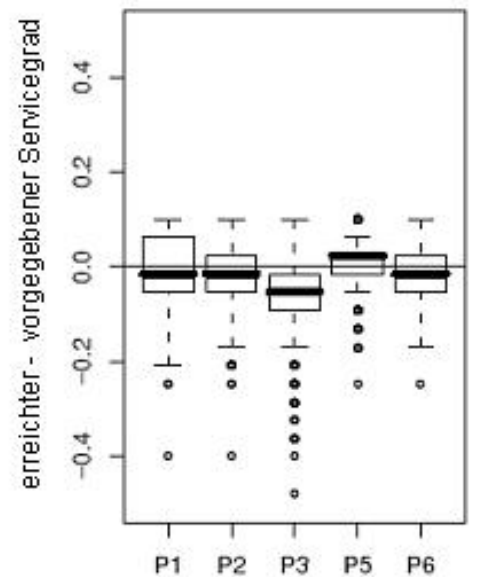

Ziel: $90 \%$

Nachfrage: gammaverteilt

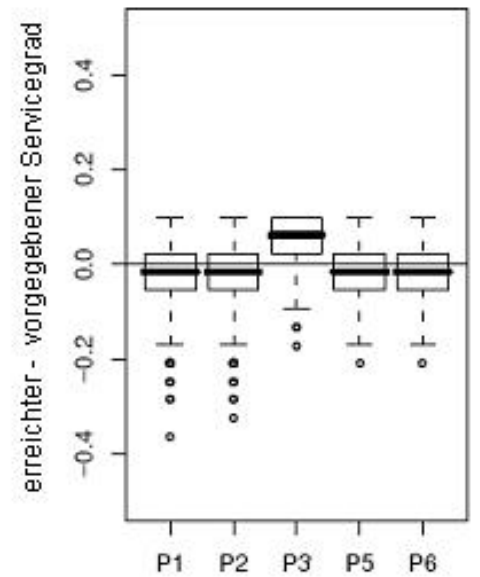

Abbildung 2: Unterschiede zwischen Ziel- und erreichtem Servicegrad, gamma- und normalverteilte Nachfrage

Der Vergleich von Ziel- und erreichten $\alpha$-Servicegraden sowie von Sicherheitsbeständen bei den verschiedenen Lagerhaltungsstrategien lässt folgende Schlüsse zu.

Zunächst betrachten wir den Einfluss der Modell- oder Verteilungsannahmen auf die erreichten Servicegrade. Weder erfüllte noch verletzte Nachfrage-Modellannahmen implizieren ein besseres oder schlechteres Ergebnis. Strategien mit unterschiedlichen Verteilungs- oder Modellannahmen führen zu unterschiedlichen erreichten Servicegraden. Aber überraschenderweise führt eine Verletzung der Annahmen nicht zwingend zu einer höheren absoluten Differenz zwischen dem erreichten und dem Zielservicegrad. Dies ist am Vergleich der Leistungen von P1 und P2 anhand der simulierten normalverteilten Daten zu erkennen. Obwohl die Strategie P2 eine Gammaverteilung unterstellt und diese Annahme verletzt ist, kommt P2 zu besseren Ergebnissen als P1, für die die Normalverteilungsannahme erfüllt ist. Auch für die simulierten gammaverteilten Daten schneidet Strategie P2 besser ab als Strategie P1. In diesem Fall erzielt die Strategie mit erfüllten Modellannahmen bessere Ergebnisse als P1, deren Modellannahmen für die Nachfrage verletzt sind. 

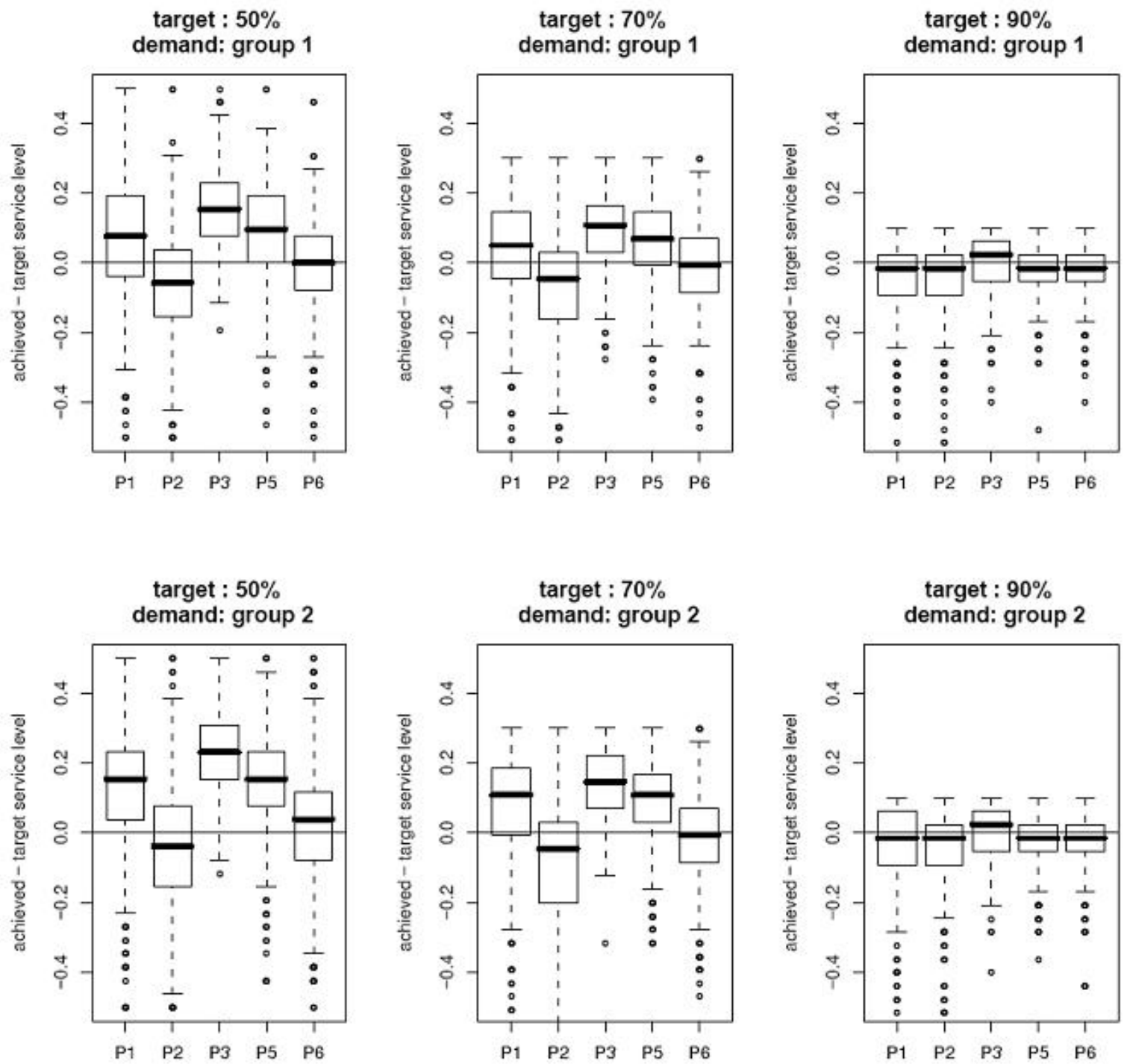

Abbildung 3: Unterschiede zwischen Ziel- und erreichtem Servicegrad, Gruppe 1 und Gruppe 2

Das zweite Ergebnis ist, dass sich die Ergebnisse mit wachsendem Zielservicegrad verbessern, dies ist der Einfluss des Zielservicegrades. Mit steigendem $\alpha$-Servicegrad werden die Unterschiede zwischen den Lagerhaltungsstrategien bezüglich der Einhaltung der gegebenen Servicegrade vernachlässigbar, sodass der Wahl einer Strategie eine geringere Bedeutung zukommt.

Drittens gibt es einen Einfluss von Nullnachfrageanteilen auf die erreichten Servicegrade. Übersteigt das Verhältnis von Nullnachfrageperioden den angestrebten $\alpha$-Servicegrad, so ist der erreichte Servicegrad größer als der Zielservicegrad. Außerdem führt Lagerhaltungsstrategie P1 bei unterstellter Normalverteilung bei steigenden Nullnachfrageanteilen zu steigendem erreichten $\alpha$-Servicegrad, selbst wenn die Nullnachfrageanteile den Zielservicegrad nicht überschreiten. Falls also die frühere Häufigkeit der Nullnachfrage eines Gutes höher ist als der gewünschte Servicegrad oder der Unterschied nur gering ist, wird der Zielservicegrad bereits durch die Nullnachfrageperioden erreicht und es ist möglicherweise unnötig, dieses Gut in Zukunft auf Lager zu halten. Diese Erkenntnisse legen nahe, zunächst die früheren Anteile von Nullnachfragen zu bestimmen und dann über die Notwendigkeit einer Lagerhaltungsstrategie zu entscheiden. 

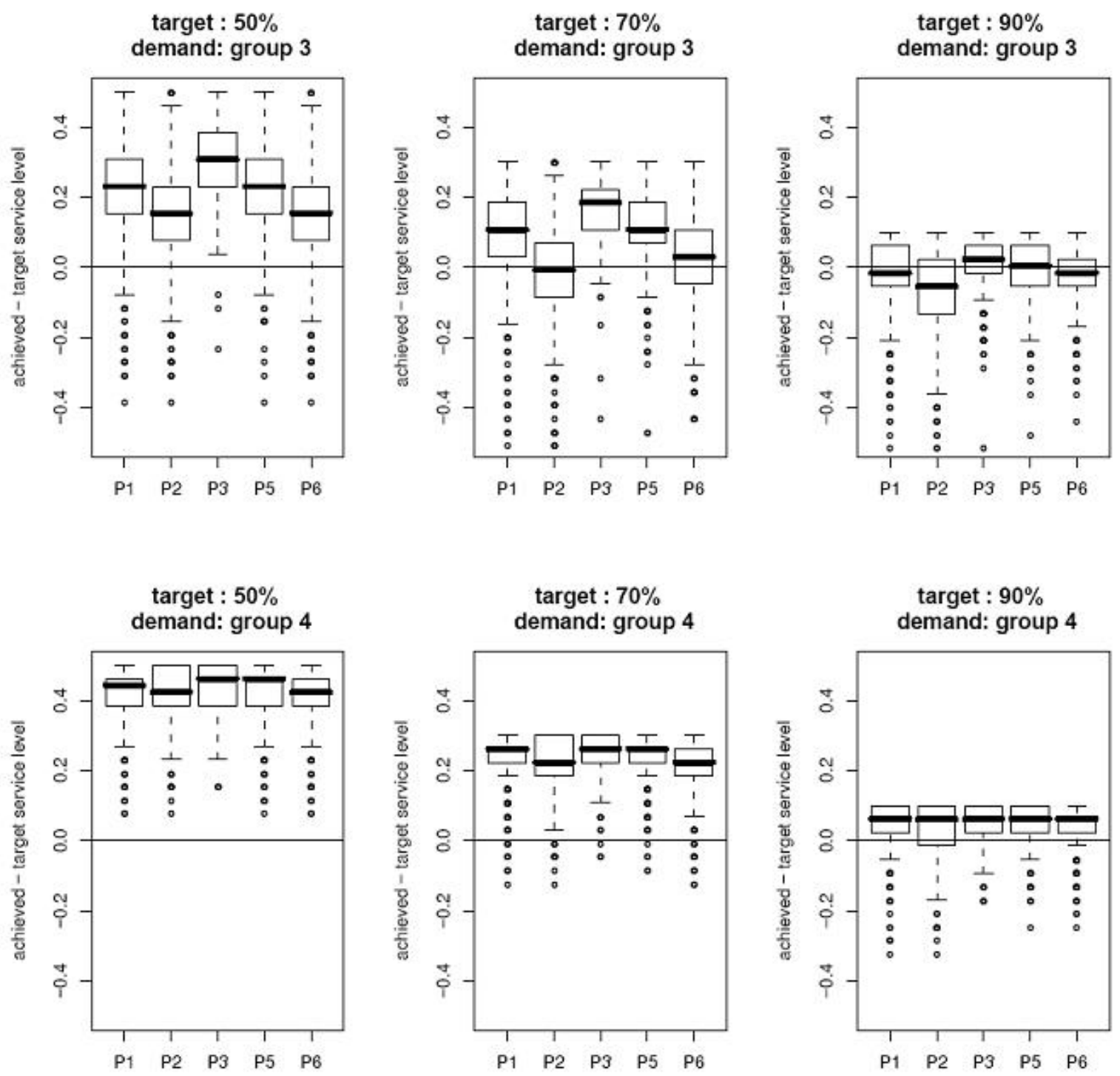

Abbildung 4: Unterschiede zwischen Ziel- und erreichtem Servicegrad, Gruppe 3 und Gruppe 4

In den Gruppen 1 bis 4 mit den realen Lagersituationen konnten die in Tabelle 2 aufgeführten Strategien den gewünschten $\alpha$-Servicegrad am besten herstellen. Soll für alle Güter eines Lagers nur eine Strategie ausgewählt werden, so ist die nichtparametrische Strategie P6 zu empfehlen.

\begin{tabular}{l|lll}
\hline & \multicolumn{3}{|l}{ Ziel- $\alpha$-Servicegrad } \\
Nachfrage & $50 \%$ & $70 \%$ & $90 \%$ \\
\hline Gruppe 1 & P6 & P6 & P6 \\
Gruppe 2 & P6 & P6 & P6 \\
Gruppe 3 & P2 oder P6 & P2 oder P6 & P3 oder P6 \\
Gruppe 4 & P1 oder P6 & P1 oder P6 & P2 oder P6 \\
\hline
\end{tabular}

Tabelle 2: Strategien mit besten erreichten $\alpha$-Servicegraden 


\section{Zusammenfassung}

In praktischen Anwendungen werden Lagerhaltungsstrategien benötigt, um automatisierte Entscheidungen über optimale Lagerbestände und Bestellmengen zu erhalten. Die meisten Lager enthalten eine große Anzahl verschiedener Artikel, so dass individuelle Entscheidungsprozesse nicht möglich sind.

In der Literatur werden verschiedene Lagerhaltungsstrategien vorgeschlagen, die sich in Lagerhaltungsmodellen und Modellannahmen unterscheiden. Für die meisten Lager kann die Gültigkeit der Modellannahmen nicht für jeden einzelnen Artikel überprüft werden. Daher werden in dieser Arbeit die Lagerhaltungsstrategien bezüglich ihrer Leistung in simulierten Situationen mit erfüllten und verletzten Annahmen bzgl. der Nachfragemodelle verglichen. Zusätzlich wird die Eignung der Strategien in einer realen Lagersituationen untersucht. Die betrachteten realen Lagerdaten weisen einen hohen Anteil von Nullnachfrageperioden auf.

Die Lagermodelle, die in dieser Untersuchung verglichen werden, enthalten Strategien mit

- u.i.v. normal- und gammaverteilter Nachfrage

- $\quad$ integrierten Moving Average Modellen der Nachfrage.

Die unterschiedlichen Modell- und Verteilungsannahmen führten zu Veränderungen der erreichten Servicegrade, aber verletzte Annahmen führten nicht zwingend zu größeren Unterschieden zwischen dem erreichten und zum Ziel gesetzten Servicegrad. Es zeigte sich, dass die erreichten Servicegrade durch die Anteile von Nullnachfrageperioden beeinflusst wurden. Der erreichte Servicegrad kann nicht kleiner sein als der Nullnachfrageanteil und daher kann der erreichte Servicegrad kaum nachgefragter Artikel größer sein als der Zielservicegrad, auch wenn die entsprechenden Artikel nicht im Lager vorhanden sind.

Ein Ergebnis dieser Studie ist, dass keins der Nachfragemodelle für alle betrachteten realen Lagersituationen als geeignet angesehen werden kann. Die aktualisierte nichtparametrische Strategie weist insgesamt die besten Ergebnisse auf. Außerdem führen weder erfüllte noch verletzte Annahmen bzgl. der Nachfragemodellannahmen zu besseren oder schlechteren Ergebnissen. Daher empfehlen wir, aktualisierte verteilungsfreie Schätzer für die Nachfrage für das Lagermanagement zu verwenden.

\section{Literatur}

[Abel. 1999] Abels, H.: DISKOVER II, Ganzheitliche Bestimmung von Sicherheitsbeständen. Berlin Heidelberg New York: Springer.

[Arro. 1951] Arrow, K.J., Harris, T., Marschak, J.: Optimal inventory policy. Econometrica 19, 250-271.

[Ceti. 2004] Cetinkaya, S., Parlar, M.: Computing a stationary base-stock policy for a finite horizon stochastic inventory problem with non-linear shortage costs. Stochastic Analysis and Applications 22, 589-625.

[Dvor. 1953] Dvoretzky, A., Kiefer, J., Wolfowitz, J.: The inventory problem: II. case of unknown distributions of demand. Econometrica 21, 450-466.

[Gall. 2004] Gallego, G., Hu, H.C.: Optimal policies for production/inventory systems with finite capacity and Markov-modulated demand and supply processes. Annals of Operations Research 126, 21-41.

[Harr. 1915] Harris, F. W.: Operations Cost. Factory Management Series, Chicago: Shaw.

[Heat. 1994] Heath, D.C., Jackson, P.L.: Modelling the evolution of demand forecasts with application to safety stock analysis in production/distribution systems. IIE Transactions 6, 17-30.

[Holl. 2002] Hollier, R.H., Mak, K.L., Lai, K.K.: Computing optimal (s,S) policies for inventory systems with a cut-off transaction size and option of joint replenishment. International Journal of Production Research 40, 3375-3389.

[Igle. 1963] Iglehart, D.L.: Optimality of $(\mathrm{s}, \mathrm{S})$ policies in the infinite horizon dynamic inventory problem. Management Science 9, 259-267.

[John. 1975] Johnson, G.D., Thompson, H.E.: Optimality of myopic inventory policies for certain dependent demand processes. Management Science 21, 1303-1307.

[Pres. 2004] Presman, E., Sethi, S.P.: Stochastic inventory models with continuous and poisson demands and discounted and average costs. Working Paper, The University of Texas at Dallas, Richardson, TX.

[Scar. 1960] Scarf, H.: Optimality of $(\mathrm{s}, \mathrm{S})$ policies in the dynamic inventory problem. Mathematical Methods in the Social Sciences. Stanford, California: Stanford 
University Press, K.J. Arrow, K. Karlin, P. Suppes, eds.

[Schn. 1981] Schneider, H.: Effect of service-levels on order-points or order-levels in inventory models. International Journal of Production Research 19, 615-631.

[Silv. 1998] Silver, E. A., Pyke, D. F., Peterson, R.: Inventory Management and Production Planning and Scheduling. New York: Wiley.

[Temp. 2006] Tempelmeier, H.: Inventory Management in Supply Networks. Norderstedt: Books on Demand.

[Tokt. 2001] Toktay, L.B., Wein, L.M.: Analysis of a forecasting-production-inventory system with stationary demand. Management Science 47, 1268-1281.

[Yu 1995] Yu, G.: Robust economic order quantity models. European Journal of Operations Research 100, 482-493. 